\title{
1. ELECCIONES Y CICLOS ELECTORALES EN ESPAÑA *
}

\author{
JOSE R. MONTERO \\ Catedrático de Ciencia Política \\ Universidad de Cádiz
}

\begin{abstract}
Este artículo recoge el texto de la conferencia pronunciada en febrero de 1987 en el Ayuntamiento de Málaga, dentro del ciclo sobre "La función de los partidos políticos en la sociedad actual». Con la excepción de algunos pequeños cambios de actualización, he preferido respetar la estructura original de la conferencia, por lo que no he modificado el tono introductorio ni incluido las tablas al uso (que pueden por lo demás encontrarse sin dificultades en la bibliografía seleccionada).
\end{abstract}




\section{SUMARIO}

Iintroducción.I. - Cuatro Elecciones legislativas, Dos Ciclos Electorales.II.-El Primer Ciclo Electoral: Elecciones Inaugurales (1977) Y Elecciones de Transicion (1979).III.-EL SEgundo Ciclo EleCtoral: ElECCIONES de "CATACLISMO" (1982) Y EleCCIONES de "Espera" (1986).IV. - Perspectivas Futuras: de NueVo, La INCERTIDUMBRE (RELATIVA). 
Revista de Derecho Político, núm. 25, 1987, págs. 9-34

\section{ELECCIONES Y CICLOS ELECTORALES EN ESPAÑA}

POR

JOSE R. MONTERO

Catedrático de Ciencia Política

Universidad de Cádiz

\section{INTRODUCCION}

Si ha habido alguna vez una "década prodigiosa" de contenido fundamentalmente político, ésa ha sido sin duda la vivida por los españoles desde 1977. Experimentábamos entonces todas las dificultades e incógnitas del recién comenzado proceso de transición desde una larga dictadura. Al cabo, formamos parte de la escasa treintena de paises cuyo sistema democrático es virtualmente irreversible. Va de suyo que las elecciones han supuesto un factor decisivo para la consecución de ese resultado, de forma similar a como los partidos y líderes políticos han desempeñado un protagonismo no menos básico. A lo largo de la "década prodigiosa", cada uno de estos elementos ha conocido un desarrollo extraordinario por muchos motivos. En la primavera de 1977 aparecía la normativa electoral que habría de regular la primera consulta democrática después de 41 años. En junio de 1987 los españoles, simultánea o sucesivamente, nacional o regionalmente, habremos contabilizado nada menos que 26 procesos electorales: dos referendums nacionales y cinco autonómicos, cuatro elecciones legislativas, tres locales y unas europeas, nueve elecciones autonómicas en las cuatro Comunidades "especiales" y otras dos en las trece restantes. Los electores vascos y andaluces han sido convocados a las urnas en catorce ocasiones, en trece los catalanes y los gallegos, en doce los restantes españoles.

Ante tamaña intensidad no resulta extraño que los partidos politicos hayan conocido todas las situaciones prácticamente imaginables de 
proliferación y desaparición, de éxito y fracaso, de coaliciones y escisiones. Cerca de 4.500 candidatos, pertenecientes a algo más de 150 partidos y coaliciones, se presentaron a las primeras elecciones legislativas ${ }^{1}$. Diez años después, las solicitudes de inscripción de partidos han superado las 500, de las que sólo la mitad aproximadamente se han inscrito en el correspondiente Registro; pero el número de los que tienen una capacidad organizativa mínima no llega a la veintena ${ }^{2}$. De los partidos de ámbito nacional representados en las Cortes de 1977 y 1979 han desaparecido los de extrema izquierda y extrema derecha, los demócratas cristianos y sobre todo la Unión de Centro Democrático (UCD); otros carecen ya de existencia por haberse fusionado (como el Partido Socialista Popular tras 1977) o haberse disuelto la coalición de la que formaban parte (como algunos de los integrantes de la Coalición Democrática después de 1979). En el mismo nivel nacional, han abundado también los casos de formación, reestructuración y disolución de coaliciones (como los de la propia UCD en 1977, Unión Nacional y Coalición Democrática en 1979, Coalición Popular [CP] en 1982 y 1986, e Izquierda Unida [IU] en 1986), así como la aparición de nuevos partidos en las Cortes, productos casi siempre de escisiones (como el Centro Democrático Social [CDS] o el Partido Demócrata Popular [PDP]). Por si esto fuera poco, los procesos de crisis sufridos por las principales organizaciones políticas han afectado incluso a sus respectivos niveles de liderazgo. Después de las elecciones de 1979, por ejemplo, ¿quién podía imaginar que los dirigentes de los partidos nacionales más importantes iban a conocer un escalonamiento de dimisiones? Lo cierto es, sin embargo, que Felipe González dimitió por unos meses de su cargo de Secretario General del PSOE en mayo de 1979, Adolfo Suárez nada menos que de la Presidencia del Gobierno en enero de 1981, Santiago Carrillo de la Secretaría General del Partido Comunista de España (PCE) en noviembre de 1982, Manuel Fraga de la Presidencia de Alianza Popular (AP) en diciembre de 1986 y Oscar Alzaga de la Presidencia y de su escaño del PDP en mayo de 1987.

LUIS ARAQUISTAIN señaló poco antes de la Dictadura de Primo de Rivera que, «en España, después de los toros, nada embriaga tanto como las elecciones" ${ }^{3}$. Cabria añadir que nunca fueron tan necesarias como en los años que siguieron a la dictadura franquista. Las tres clásicas funciones que las elecciones suelen cumplir en cualquier sistema democrático (las de producir representación, crear gobiernos y reforzar la

1 Francisco Gonzalez Ledesma y otros, Las elecciones del cambio. Barcelona. Plaza y Janés, 1977, pág. 215; y José María Maravall, La política de la transición. Madrid: Taurus, 2. a ed., 1984, pág. 36.

2 Michael BUSE, La nueva democracia española. Sistema de partidos y orientación del voto (1976-1983). Madrid. Unión Editorial, 1984, págs. 28 y 135 y ss.

3 Luis Araquistán, España en el crisol: Un Estado que se disuelve y un pueblo que renace. Barcelona. Minerva, circa 1920; citado por Amando DE MIGUEL, El rompecabezas nacional. Barcelona. Plaza y Janés, 1986, pág. 133. 
legitimidad) se cumplieron en grados extraordinariamente altos durante la pasada década. Gracias a las numerosas, para muchos excesivas, consultas celebradas, los españoles han sido capaces de completar la transición política pacíficamente y sin demasiados costes, ratificar la Constitución más duradera de nuestra historia, consolidar un nuevo sistema democrático con altas dosis de legitimidad, realizar una alternancia en el Gobierno de modo casi plebiscitario, transformar los equipos de gobierno de todos los municipios, sustituir las viejas estructuras estatales centralistas por las del Estado de las Autonomías e, incluso, participar en los destinos comunitarios mediante la elección de sus representantes en el Parlamento europeo. Es cierto que tan nutrida cantidad y variedad de procesos electorales no han podido por menos que mostrar a veces disfunciones notables sobre, por ejemplo, las restricciones de la representación, las relaciones de los partidos con la sociedad civil o las consecuencias negativas de la personalización de la vida política a través de los líderes. Estoy convencido, sin embargo, de que el balance, al menos hasta el momento, es abrumadoramente positivo. Lo que sigue intentará sistematizar los que en mi opinión son aspectos fundamentales de la pasada década electoral. Para ello me limitaré a los de las cuatro elecciones legislativas (aunque haciendo hincapié en las dos últimas) y a los partidos relevantes de ámbito nacional. Pese a las muchas consultas y partidos de importancia que se quedan fuera (sobre todo, las autonómicas y los nacionalistas, respectivamente), la naturaleza de este trabajo introductorio no permite más que señalar los hitos significativos, subrayar ciertas cuestiones básicas, plantear algunos problemas irresueltos.

\section{CUATRO ELECCIONES LEGISLATIVAS, DOS CICLOS ELECTORALES}

Los estudios electorales suelen abordar las consultas legislativas nacionales desde dos ópticas diferentes. De un lado, los análisis que piensan que cada elección es única e irrepetible, tienen su pequeña historia propia y arrojan unos resultados peculiares. De otro, los que observan ante todo la reiteración de situaciones comunes y enfatizan los elementos de continuidad en una serie más o menos larga de elecciones. Aunque es probable que el enfoque más fructífero sea el que combine ambas perspectivas, como luego intentaremos comprobar, las elecciones españolas contienen el suficiente número de facetas para permitir su consideración desde cada una de aquéllas. Así, por ejemplo, los partidarios de la continuidad han solido argüir la constante de sus resultados «bipartidistas» a pesar del cambio de partidos (UCD y Partido Socialista Obrero Español [PSOE] en 1977-1979, y PSOE y CP en 1982-1986), una conclusión no por errónea menos frecuente en niveles periodísticos, políticos y 
académicos. Los defensores de la especificidad, por su parte, apuntan a su favor las extraordinarias circunstancias que en mayor o menor medida han concurrido en todas y en cada una de las elecciones celebradas. Las de 1977 serían excepcionales por su doble carácter inaugural del sistema de partidos y definitorio del tipo de transición política; las de 1979, por los intentos del PSOE para sustituir a UCD y el empeño de UCD para revalidar su mandato; las de 1982, por el triunfo del PSOE, la desaparición virtual de UCD y la magnitud de los procesos de cambio de voto; y las de 1986 , en fin, por la victoria socialista al conseguir de nuevo una comparativamente rara mayoría absoluta de escaños y por los movimientos estratégicos de los partidos de centro-derecha para constituirse en alternativa al PSOE.

Las dos perspectivas citadas pueden integrarse conjuntamente en un enfoque que agrupe las cuatro elecciones legislativas en dos ciclos ${ }^{4}$. En el primero se comprenderían las de 1977 y 1979; en el segundo, las de 1982 y 1986 . Uno y otro comparten diferencias y similitudes que avalarían su distinción. La mayor parte de las diferencias son tan evidentes como conocidas. Del primer ciclo han emergido gobiernos homogéneos pero minoritarios de centro-derecha, cuyo grupo parlamentario, la UCD, ha tenido en consecuencia que buscar apoyos ocasionales para su labor legislativa. El segundo ciclo, en cambio, comprende elecciones de mayoría absoluta de escaños, lo que ha posibilitado la formación de gobiernos homogéneos del PSOE y facilitado sobremanera su tarea parlamentaria. El primer ciclo dio nacimiento a un sistema pluripartidista moderado sobre cuya larga duración existía un acuerdo generalizado; el segundo, a un sistema de partido dominante que por ello fue estimado absolutamente provisional. Como consecuencia de sus derrotas electorales en el primer ciclo, el PSOE supo sacar provecho de las debilidades de su rival y elaborar una estrategia acorde con su objetivo de llegar al Gobierno. Todo lo contrario de lo ocurrido con AP, cuya lectura de los resultados de 1982 le llevó a formular planteamientos de oposición erróneos y a aplicar una estrategia de acceso al Gobierno tan irreal como errática: la ruptura de Coalición Popular y la grave crisis sufrida por AP fueron sus inmediatas consecuencias.

Los dos ciclos que estamos considerando conocieron también algunas similitudes dignas de mención. Cada uno de ellos está compuesto por una elección «excepcional» (1977 y 1982, respectivamente) y por otra "normal» u «ordinaria" (1979 y 1986). Los motivos de su respectiva excepcionalidad son evidentes. En 1977 se inauguraban los mecanismos electorales democráticos tras una larga dictadura, se ratificaba el inicio de

4 Utilizo el término ciclo en su acepción más genérica, ni siquiera coincidente con el significado que se le otorga en la literatura electoral; cfr. Cees VAN DER EIJK, "Testing theories of electoral cycles", en European Journal of Political Research, 15. 1987, págs. 253 y ss. 
la transición, se abria una etapa constituyente, se daba nacimiento a un sistema competitivo de partidos. $Y$ en 1982 se expresaba con el voto el realineamiento del sistema de partidos en proporciones considerables, una condena tácita del golpismo y la consolidación definitiva de la democracia, y el acceso al Gobierno, por vez primera tras más de cuarenta años, de un partido de izquierdas. Al lado de estas extraordinarias dimensiones, las elecciones de 1979 y 1986 no podian por menos que aparecer como "normales» o "rutinarias". De ahí la alta participación registrada en las elecciones "excepcionales" y el crecimiento del abstencionismo en las "normales", en una pauta de variaciones bruscas de la participación electoral que hace del español un caso único entre los países europeos ${ }^{5}$. Y de ahí también la sorpresa de los analistas al comprobar la continuidad básica de los resultados de cada elección «normal» con su respectiva precedente, cuando precisamente el carácter «excepcional» de las de 1977 y 1982 apostaba por su condición de irrepetibles. Cabe señalar asimismo que cada ciclo electoral ha conocido el fracaso del principal partido de la oposición para sustituir al Gobierno, bien que el estrépito del experimentado por CP en 1986 no sea parangonable con el del PSOE en 1979. Y que cada ciclo ha observado sendos intentos de creación de partidos con vocación de "bisagra" entre los dos principales: unos intentos que se quedaron en eso en 1979 por la nauraleza non nata de un Partido Radical que habría de reorientar la mayoria parlamentaria entre UCD y PSOE, y unos intentos que en 1986 se dirigieron hacia el espacio politico de centro existente entre el PSOE y la CP obteniendo por el momento los resultados modestos del CDS o saldándose con el fracaso sin paliativos del Partido Reformista Democrático (PRD).

¿Qué trazos comunes laten tras las divergencias y similitudes de estos dos ciclos? Aunque la celebración de sólo cuatro elecciones legislativas no permita ser demasiado concluyentes, ¿qué pautas parecen haber caracterizado el comportamiento electoral de los españoles durante la pasada década, y cómo han repercutido sobre la evolución de los partidos? Dejando al margen, como ya se dijo, el voto y los partidos nacionalistas (cuya presencia resulta obviamente decisiva sobre todo en las Comunidades vasca y catalana), las principales pautas que merecen señalarse son, en mi opinión, las siguientes:

1. La simplificación del mapa partidista ha ido acentuándose de consulta en consulta. No es sólo que en la primera se redujera drásticamente la "sopa de siglas» a unas pocas significativas, sino que el porcentaje de votos y escaños acumulados por los dos primeros partidos (UCD y PSOE en 1977-1979; PSOE y CP en 1982-1986) ha crecido pau-

5 José Ramón MONTERO, "Elecciones "normales" y elecciones "excepcionales": Algunos datos y factores de la movilización electoral de octubre de 1982", en el volumen colectivo Homenaje a Carlos Ruiz del Castillo. Madrid. Instituto de Estudios de Administración Local, 1985 , págs. 415 y ss. 
latinamente. Los índices de fragmentación electoral y parlamentaria, que en 1977 eran de los más elevados entre los países europeos, han experimentado la correspondiente disminución ${ }^{6}$. A ello han colaborado la canalización de las preferencias de los electores y la combinación de sus "votos útiles" y "votos de castigo", así como el funcionamiento de la normativa electoral ${ }^{7}$.

2. Junto con Grecia y Portugal, España personificó una excepción en el marco de las democracias occidentales en dos aspectos complementarios de gran importancia. De un lado, el realineamiento electoral español de 1982 rompió la tendencia por la que la distribución de las preferencias de los votantes en las primeras elecciones se mantiene constante durante algunas décadas; así había ocurrido en países tan diversos como Francia, Finlandia, Alemania, Italia y Japón. De otro lado, las jóvenes democracias del Sur de Europa han conseguido realizar en muy poco tiempo la alternancia en el Gobierno, una situación que ha necesitado muchos más años en algunos países y que en otros (como Italia y Japón) todavía no se ha producido ${ }^{8}$.

3. La mayor parte de los partidos relevantes se han visto sacudidos por crisis de distinta naturaleza: para unos han sido de adaptación, institucionalización o crecimiento tras su irrupción en la legalidad democrática; para otros, crisis derivadas de conflictos personales o de luchas faccionales en el nivel de sus élites; y aún para otros a causa del enfrentamiento excluyente de modelos a los que se quería adaptar el partido ${ }^{9}$. Como cabía esperar, estas crisis han incidido en los resultados electo-

6 Antonio BAR, «El sistema de partidos en España: Ensayo de caracterización», en Sistema, 47, 1982, págs. 8-9; del mismo, «¿Normalidad o excepcionalidad?: Para una tipología del sistema de partidos español, 1977-1982", en Sistema. 65, 1985, págs. 14 y ss.; y Josep M. VALLÉS, «Diez años de elecciones democráticas en España (1977-1987): Una visión panorámica", de próxima publicación en Zeitschrift für Parlamentsfragen, págs. 3-4.

7 Mario CACIAGLI, Elecciones y partidos en la transición española. Madrid. Centro de Investigaciones Sociologicas/Siglo XXI, 1986, págs. 78 y ss.; Josep M. VALLÉs, Reforma electoral i coordenades políticas. Barcelona. Fundació Jaume Bofill, 1982; del mismo, "Sistema electoral y democracia representativa: Nota sobre la Ley Orgánica del Régimen Electoral General de 1985 y su función política", en Revista de Estudios Políticos, 53, 1986, págs. 7 y ss.; y Giacomo SANI y Richard GUNTHER, "¿Qué hubiera pasado si...?: El Impacto de la normativa electoral', en Juan J. Linz y José R. MONTERo (eds.), Crisis y cambio: Electores y partidos de la España de los años ochenta. Madrid. Centro de Estudios Constitucionales, 1986, págs. 125 y ss.

\& Guillermo O'DONNELl y Philippe C. SCHMITTER, Transitions from authoritarian rule. Tentative conclusions about uncertain democracies. Baltimore. Johns Hopkins University Press, 1986, págs. 62 y ss.

9 Richard GUNTHER, “Models - and crises- of spanish political parties», en Juan J. LINZ y R. GUNTHER (eds.,), Politics and society in the new democratic Spain (de proxima publicación en Berkeley. University of California Press); del mismo, "Democratization and party building: Contradictions and conflicts facing party elites in the spanish transition to democracy", en Robert P. CLARK (ed.), Spain in the 1980 (de próxima publicación en Cambridge, Mass. Ballinger). 
rales de los partidos de modo distinto: positivo a corto plazo para el PSOE y a largo término para la AP de 1978, negativo para el PCE, catastrófico para UCD. Queda todavía como problema pendiente, sin embargo, el de la articulación política de la derecha, un problema que tiene profundas raices históricas, que se resolvió sólo provisionalmente durante la transición y al que la penúltima crisis de AP tras las elecciones de 1986 ha conferido una renovada trascendencia.

4. La inestabilidad mostrada por el sistema de partidos español no ha impedido afortunadamente la progresiva estabilización de su sistema democrático. Contra la opinión de algunos autores, la estabilidad del sistema partidista no ha sido ni la consecuencia ni un requisito para la estabilidad democrática. Como se ha escrito, "esto indica que los españoles no contemplan en el horizonte alternativa alguna a la democracia y que han llegado a un consenso básico a la hora de valorar las instituciones democráticas como esenciales para una vida civil y para la participación en el inundo occidental, y por lo tanto, como valiosas en sí mismas» ${ }^{10}$. El cambio drástico sufrido por el sistema de partidos no ha parecido tener incidencias negativas en las opiniones sobre la legitimidad de la democracia. Por el contrario, la llegada del PSOE al Gobierno tuvo efectos claramente positivos sobre las reservas de legitimidad y eficacia del sistema, contribuyendo asi a aumentar los niveles de estabilidad democrática $^{11}$

5. La inestabilidad del sistema de partidos contrasta además con la estabilidad básica mostrada por las actitudes y orientaciones políticas de los españoles. Los electores no produjeron el realineamiento de 1982 al votar a partidos distintos de los que lo habian hecho en 1979 como consecuencia de una modificación de sus actitudes, sino porque los partidos a los que habían votado antes no eran los mismos en 1982. En palabras de SANTAMARÍA, "la exacerbada volatilidad de 1982 no indica que el electorado estuviera plenamente "desestructurado" y "disponible". Fue más bien la desestructuración y la reestructuración del sistema de partidos previa a las elecciones lo que, unido a una serie de cambios en el entorno, puede explicar aquel nivel de volatilidad" ${ }^{12}$. Ha podido enfatizarse de esta forma la contradicción entre «partidos volátiles y electores pág. 660.

10 LINZ, "Consideraciones finales", en Linz y MONTERo (eds.), Crisis y cambio, cit.,

11 José María Maravall y Julián Santamaria, "Crisis del franquismo, transición politica y consolidación de la democracia en España", en Sistema, 68-69, 1985, págs. 128-129; y J. SANTAMARIA, "Elecciones generales de 1982 y consolidación de la democracia», en $R e-$ vista Española de Investigaciones Sociológicas, 28, 1984, págs. 9 y ss.

12 Santamaria, "Elecciones generales de 1982", cit., pág. 14. También, Richard GUNTHER, "Un análisis preliminar de las alteraciones producidas en 1982 en el sistema de partidos", en Revista de Estudios Políticos, 45, 1985, págs. 7 y ss.; y del mismo, "El realineamiento del sistema de partidos de 1982", en LINZ y MONTERo (eds.), Crisis y cambio, cit., págs. 27 y ss. 
estables» ${ }^{13}$, una situación que, por lo demás, se presenta en el reverso de la observada tradicionalmente en los sistemas europeos.

6. La volatilidad de las opciones partidistas se debe en parte a la modernidad del sistema democrático español. En cuanto fruto tardío, las relaciones entre los partidos y sus votantes no estaban condicionadas por una estructura rigida de clases sociales, ni mediadas por una subcultura ideológica transmitida intergeneracionalmente, ni necesitadas de redes capilares de militantes para desarrollar sus actividades ${ }^{14}$. Ausentes estos requisitos, el nacimiento de un sistema de partidos en una época de financiación pública y de utilización masiva de los medios de comunicación, fundamentalmente de la televisión, ha contribuido a personalizar el liderazgo, a debilitar el arraigo social de los partidos, a amortiguar sus diferencias programáticas ${ }^{15}$. Como ha señalado LINZ, «la importancia atribuida a los líderes y a su personalidad frente a la ideología e incluso al programa - dentro de ciertos límites que excluyen posiciones extremas-, y la misma existencia del voto útil, son resultados de esa "modernidad" del sistema democrático español. En una cultura ideológica, de fidelidades a posiciones ideológicas definidas y de lealtades institucionales, asociativas, organizativas e incluso personales y clientelísticas, el voto útil nunca hubiera tenido tanta importancia, y la volatilidad electoral hubiera sido menor " ${ }^{16}$. Los bajos niveles de identificación partidista y los escasos recursos organizativos de los partidos se convierten asi en condiciones necesarias pero no suficientes para dar cuenta de los procesos de cambio de voto. En ese contexto (hasta cierto punto inevitable dada la coyuntura histórica de la transición), adquieren mucha mayor relevancia las respuestas de los electores ante los problemas presentes en cada elección, la fiabilidad de los lideres llamados a resolverlos y las imágenes de capacidad y responsabilidad de los componentes de la estructura de partidos $^{17}$.

7. Finalmente, la evolución electoral de los partidos ha estado presidida por la moderación de los ciudadanos, una característica que ha permanecido asimismo constante durante la pasada década. Numerosos estudios han demostrado que esta moderación, que ejerció un poderoso efecto centrípeto sobre las estrategias electorales de los partidos, se manifestó en al menos cuatro formas. Primera, en las preferencias de los españoles sobre las políticas de cambio social y político, lo que les hacia

13 Samuel H. Barnes, Peter MCDONOUgh y Antonio Lopez Pina, "Volatile parties and stable voters in Spain", en American Journal of Political Science, 29, 1985, págs. 56 y ss.

14 Josep M. VALLES, "A la busca de un sistema: Los partidos políticos en España", en Razón y Fe, 1060, 1987, págs. 143-144.

15 José Ramón MONTERO, "Partidos y participación política. Algunas notas sobre la afiliación política en la etapa inicial de la transición española", en Revista de Estudios Politicos, 23, 1981, págs. 64 y ss.

16 LINZ, "Consideraciones finales", cit., pág. 659.

17 Maravall y Santamaria, "Crisis del franquismo", cit., págs. 121-123. 
comparables a los ciudadanos de las restantes sociedades europeas. Segunda, en la distribución unimodal del electorado en la escala ideológica izquierda-derecha, con una concentración sustancial en las posiciones de centro y centro-izquierda. Ello ha permitido el acceso al Gobierno a partidos de centro en sentido amplio: en el primer ciclo de centro-derecha, la UCD, y en el siguiente de centro-izquierda, el PSOE; y ello dificulta, dicho sea de paso, que la alternativa al PSOE pueda radicar en un partido claramente de derechas como AP. Tercera, en el cada vez más escaso apoyo electoral otorgado a los partidos extremos o concebidos como tales. Y, cuarta, en la misma continuidad básica de todos estos indicadores; una continuidad demostrativa de que esa moderación, más que deberse a la expresión provisional de unos ciudadanos temerosos o sujetos a un proceso fluido de cambios actitudinales, se hallaba profundamente enraizada en la sociedad ${ }^{18}$. El análisis específico de los dos ciclos electorales que hemos diferenciado nos permitirá comprobar cada uno de estos supuestos.

\section{EL PRIMER CICLO ELECTORAL: ELECCIONES INAUGURALES (1977) Y ELECCIONES DE TRANSICION (1979)}

Podemos considerar con cierta brevedad las elecciones del primer ciclo: pertenecen ya a la historia. Las de 1977 fueron, además, rigurosamente históricas por las razones ya apuntadas. Como todas las elecciones inaugurales (las que los anglosajones denominan founding elections) de los países en transición, las españolas fueron vividas con los altos niveles de entusiasmo, compromiso y participación que les caracterizan ${ }^{19}$. Convertidos en "héroes" colectivos, las decenas de partidos en liza avivaban la incertidumbre del resultado con la incógnita del comportamiento electoral de los españoles. Cuanto éste se produjo el 15 de junio, sólo cinco partidos de ámbito nacional y sólo dos regionales consiguieron representación en el Congreso ${ }^{20}$. Entre aquéllos, la UCD, con

\footnotetext{
18 - Cfr. por todos Maravall, La política de la transición, cit., págs. 32 y ss.

19 O'DONNELL y SCHMITTER, Transitions from authoritarian rule, cite., págs. 57 y ss.

20 Cfr. CaClaGLI, Elecciones y partidos de la transición española, págs. 39 y ss.; Equip. de Sociología Electoral, Sobre les eleccions legislatives del 1977. Barcelona. Estudis Electorals, 1978; Juan J. LINZ, "Il sistema partitico spagnolo", en Rivista Italiana di Scienza Politica, 8, 1978, págs. 363 y ss., y del mismo, "The new spanish party system", en Richard Rose (ed.), Electoral participation. A comparative analysis. Londres. Sage, 1980, págs. 101 y ss.; Josep M. VALLÉS, “Distribució territorial del vot a Espanya entre 1977 i 1982: Un comentari de geografía electoral", en Estudis Electorals/7. El comportament electoral a l'Estat espanyol (1977-1982). Barcelona. Fundació Jaume Bofill/Edicions de la Magrana, 1984, págs. 13 y ss.; Miguel MARTINEZ CuADRADO, El sistema político español y el comportamiento electoral regional en el sur de Europa. Madrid. Instituto de Cooperación Intercontinental, 1980, págs. 70 y ss.; y Jonathan STORY, «Spanish political parties before and after the election", en Government and Opposition, 12, 1977, págs. 62 y ss.
} 
cerca del 35 por 100 de los votos, y el PSOE, con cerca del 30 por 100 , sumaban el 81 por 100 de los escaños. Cada uno de ellos estaba flanqueado por sendos partidos con apoyos mucho más bajos: el PCE a la izquierda del PSOE, con el 9 por 100 de los votos, y AP a la derecha de UCD, con el 8 por 100. Los de extrema derecha obtuvieron menos del 1 por 100, y los de la extrema izquierda, aunque triplicaron ese porcentaje, quedaron sin representación parlamentaria. Como también les ocurrió a los demócratas cristianos, en consecuencia a una equivocada estrategia electoral y a una campaña llena de errores ${ }^{21}$. El electorado quedaba así dividido casi por mitades entre izquierda y (con un ligero predominio) derecha, y con una configuración simétrica en cada bloque. Al decir de SAN. TAMARÍA, «la significación política de los resultados era transparente. Un electorado, agrupado principalmente en torno a las opciones moderadas de derecha e izquierda, se pronunciaba inequívocamente en favor del cambio. La izquierda no conseguía el respaldo suficiente para dirigirlo, pero sí el necesario para evitar que se frenase. La desautorización a la derecha franquista impedía, por otra parte, esa posibilidad» ${ }^{22}$. En el balance general de victorias y fracasos, las mayores dosis de derrota se acumularon sobre una AP percibida como franquista; la mayor decepción, en un PCE que confiaba obtener rendimientos superiores a su oposición antifranquista. Y mientras que el PSOE aparecía como el gran vencedor de la consulta a la vista de su superioridad sobre el PCE y de su proximidad a UCD, la victoria de ésta podía ser considerada como un relativo fracaso por su incapacidad para obtener mayor provecho aún de la normativa electoral, de la escasa implantación de los partidos competidores y del apoyo popular a su estrategia reformista ${ }^{23}$.

Las elecciones de 1977 pusieron de manifiesto unas relaciones complejas de discontinuidad con respecto al período republicano. En el plano del liderazgo, la composición de la nueva élite política de 1977 supuso una brusca ruptura con la de los años treinta, con alguna excepción, como la de S. Carrillo. En el ámbito partidista, sólo el PSOE y el PCE podian presumir de una cierta continuidad, pero a costa de grandes esfuerzos de adaptación. La influencia anarquista fue sustituida, al parecer, por el voto socialista, y los partidos de centro-izquierda republicanos carecieron de herederos directos. La derecha española tuvo que acudir a dos organismos de nuevo cuño (UCD y AP) ante la incapacidad de la

21 Javier TUSELL, «The Democratic Center and Christian Democracy in the elections of 1977 and 1979 ", en Howard R. PEnNIMAN y Eusebio M. MuJAL-LeON (eds.), Spain at the Polls, 1977, 1979 and 1982. A study of national elections, Washington, D.C. American Enterprise Institute, 1985, págs. 55 y ss.; y LINZ, «ll sistema partitico spagnolo», cit., págs. 395 y ss.

22 Julián SANTAMARIA, "Transición controlada y díficultades de consolidación: El ejemplo español», en J. SANTAMARÍa (comp.), Transición a la democracia en el sur de Europa y América Latina. Madrid. Centro de Investigaciones Sociológicas, 1982, pág. 403.

23 José Ignacio WERT, “El electorado de UCD: Una versión sociológica», ponencia presentada al congreso de la Asociación Española de Ciencia Política, Zaragoza, marzo de 1983, pág. 2. 
CEDA de mantener la continuidad organizativa lograda en Italia con el PPI-DC y en Alemania con el Zentrum-CDU/CSU ${ }^{24}$. Pese a todo ello, resulta ciertamente sorprendente que en 1977 los partidos nuevos (como UCD) o renovados (como el PSOE) hayan podido contar con la lealtad de ciertos sectores sociales y geográficos que ya la habían expresado respectivamente a la derecha y a la izquierda en los últimos años de la Segunda República. MARAVALL ha hecho de la "memoria política» el factor que ha permitido la supervivencia de las lealtades socialistas en sectores sociales $\mathrm{o} / \mathrm{y}$ geográficos determinados ${ }^{25}$; la hipótesis vale también para las tendencias conservadoras o derechistas ${ }^{26}$. En suma, los cambios de todo tipo habidos durante el franquismo no impidieron la supervivencia de lealtades hacia la izquierda o la derecha tras cuarenta años de dictadura; unas lealtades que fueron transmitidas intergeneracionalmente a través de familias y comunidades. Dentro de cada una de aquéllas, la opción por un partido concreto debió ser realizada, como en otros supuestos, por razones de índole práctica (el "voto útil») o de proximidad ideológica del partido con el votante 27 .

Aunque no pocos analistas se mostraban convencidos de que la reformulación de las estrategias de los partidos acarrearía cambios inmediatos, las elecciones legislativas celebradas en marzo de 1979 confirmaron básicamente los resultados de $1977^{28}$; las únicas novedades parecieron radicar en un espectacular crecimiento del abstencionismo (más de diez puntos porcentuales) y en la llegada al Congreso de nuevos partidos regionalistas o nacionalistas. El principal beneficiario de la nueva consulta fue UCD, y no tanto por las pocas décimas de porcentaje del voto ganadas cuanto por el fracaso socialista de sustituirlo en el Gobierno. EI PSOE consiguió aumentar sólo un punto porcentual de su voto, pero se hizo más fuerte en las áreas rurales. Ambos partidos mantuvie-

24 LINZ, «ll sistema partitico spagnolo», cit., págs. 366 y ss.

25 Cf. José María Maravall, Dictadura y disentimiento político. Obreros y estudiantes bajo el franquismo. Madrid. Alfaguara, 1978, y La política de la transición, cit., págs. 39 y ss.

${ }_{26}$ Cfr. José Ramón MONTERO, “Sobre los supuestos de continuidad y discontinuidad de la derecha", en el volumen colectivo Estudios sobre la historia de España. Homenaje a Manuel Tuñón de Lara. Madrid: Universidad Internacional Menéndez y Pelayo, 1981, vol. III, págs. 223 y ss.

${ }_{27}$ Maravall, La política de la transición, cit., pág. 44; y Dario VILA CarRo y otros, "Sociología del actual cambio político en España», en Fundación FOESSA, Sintesis actualizada del III Informe FOESSA. Madrid. Euramérica, 1978, págs, 718 y ss.

28 Cfr. Richard GunTHER, Giacomo SANI y Goldie SHABAD, El sistema de partidos en España. Génesis y evolución. Madrid. Centro de Investigaciones Sociológicas/Siglo XXI, 1986; Jorge DE EstebAn y Luis LOPEZ GueRRA (eds.), Las elecciones legislativas del 1 de marzo de 1979. Madrid. Centro de Investigaciones Sociológicas, 1979; CACIAGLI, Elecciones y partidos en la transición española, cit., págs. 122 y ss.; Juan J. LiNz y otros, Informe sociológico sobre el cambio político en España, 1975-1981. Madrid. Euramérica, 1981; BAR, «EI sistema de partidos en España", cit., págs. 10 y ss.; y José María MARAVALL, "Political cleavages in Spain and the 1979 general election", en Government and Opposition, 11 (1979), págs. 118 y ss. 
ron, pues, la distancia que les separaba en 1977, así como las dos terceras partes del voto combinado y algo más del 80 por 100 de los escaños. En la izquierda, el PCE vio reproducida muy a su pesar su posición de inferioridad con respecto al PSOE, que había estimado provisional en 1977: las esperanzas por transplantar el modelo italiano de presencia comunista comenzaban a difuminarse tras los esfuerzos realizados para legitimarse y atraerse a sectores significativos del electorado socialista. En la derecha, Coalición Democrática, en la que participaba mayoritariamente AP, se llevó otra vez la palma de las derrotas. No sólo fue la única fuerza política que disminuyó sus porcentajes de voto (cerca de tres puntos porcentuales) y su número de escaños (a casi la mitad), sino que además sus desmesuradas expectativas de recuperación de posiciones a costa de UCD, y mediante una estrategia deliberada de moderación, se saldaron con un nuevo fracaso electoral ${ }^{29}$.

La continuidad básica en 1979 de la distribución de fuerzas existente dos años antes reprodujo los altos niveles de fragmentación electoral y parlamentaria; aunque cabe observar un ligero descenso de ambos índices, seguian siendo aún superiores a los de otros países europeos. También ocasionó una distribución similar de los partidos en las áreas de izquierda y derecha, si bien en esta ocasión los efectos combinados de pérdidas y ganancias permitió al voto de la izquierda exhibir una ligera superioridad. En fin, la volatilidad fue muy escasa, menor en todo caso de la que al poco tiempo se produjo con respecto a las elecciones locales y a las autonómicas del País Vasco y Cataluña. Un examen de los procesos de transferencia de votos entre los principales partidos de ámbito nacional revela un alto grado de cristalización de los espacios políticos y una relativa fluidez de los alineamientos Con la excepción notable de Coalición Democrática, las escasas transferencias que pueden constatarse no se dieron tanto entre los partidos de izquierda a los de derecha o viceversa, sino en el interior de cada bloque, y con una cierta tendencia hacia los mayores partidos más próximos al centro que a la inversa ${ }^{30}$.

Las elecciones de 1979 sivieron asimismo para confirmar otros aspectos sustanciales de la competición partidista que ya se habían apuntado con anterioridad. Para empezar, los gobiernos casi mayoritarios de UCD prefirieron seguir buscando en minorías alternativas de la derecha o de la izquierda el respaldo parlamentario suficiente. Frente a las por entonces habituales solicitudes comunistas para formar un «gobierno de concentración", la coalición entre UCD y PSOE hubiera podido generar una dinámica centrífuga de peligrosas consecuencias; de otra parte, la coalición UCD-AP resultaba inviable por sus diferencias internas y por

29 José R. MONTERo “Alianza Popular, Coalición Democrática y Coalición Popular (1976-1986): La evolución de la derecha española en la primera década democrática», en Julián SANTAMaría (ed.), Los partidos políticos españoles (de próxima publicación en Madrid. Centro de Investigaciones Sociológicas).

30 Santamaría, "Transición controlada y dificultades de consolidación», cit., pág. 413. 
la imagen autoritaria, o en cualquier caso, escasamente democrática, de $A P^{31}$. En segundo lugar, la reducción del número e intensidad de los conflictos históricos que estallaron en el periodo republicano; una reducción que se convirtió en definitiva tras el éxito de las élites políticas al conseguir la aprobación de una Constitución redactada mediante procedimientos consensuales ${ }^{32}$. La secularización y la progresiva aceptación de la Monarquía eliminaron los tradicionales conflictos religioso e institucional, y limitaron los ejes de la competencia partidista al ideológico de la izquierda-derecha y, en el marco regional, el más amplio centralismo-nacionalismo. En tercer lugar, la distribución unimodal de los ciudadanos en la escala izquierda-derecha, con porcentajes muy escasos situados en ambos extremos de la escala y los mayoritarios concentrados en sus espacios centrales, sobre todo en el centro-izquierda, forzaban a los partidos a realizar una competencia centripeta si querian mantener o/y ampliar sus electorados. Este decisivo supuesto estratégico se ejemplificó a la perfección durante la campaña electoral de 1979. Todos los partidos relevantes compitieron con los más próximos a ellos en dirección al centro, sin preocuparse de los que se hallaban alejados a su izquierda o a su derecha: el PCE lo hizo con el PSOE y no con la extrema izquierda, el PSOE con UCD y no con el PCE, UCD con el PSOE y no con Coalición Democrática, y ésta con UCD y no con la extrema derecha ${ }^{33}$. Por último, el sistema de partidos resultante no había llegado a cristalizar, como temieron algunos analistas, en un sistema multipartidista polarizado, de tendencias centrífugas, con oposiciones bilaterales y con la presencia de partidos antisistema. Se trataba, por el contrario, de un sistema pluripartidista moderado, de tendencias centrípetas según hemos señalado, con unos niveles de polarización menores a los existentes en las democracias del Sur de Europa y con la presencia en su extremos de los partidos que, como el PCE y AP, resultaban dificilmente catalogables en la categoria de antisistema ${ }^{34}$. Es cierto que este sistema se correspondia adecuadamente a la moderación política mostrada por la sociedad española. Pero

31 Maravall y Santamaría, «Crisis del franquismo», cit., págs. 96-97; Linz, “ll sistema partitico spagnolo», cit., págs. 382 y SS.; BAR, “El sistema de partidos en España”, cit., págs. 35 y ss.; y José R. MONTERO, «Los fracasos politicos y electorales de la derecha española: Alianza Popular, 1976-1986", de próxima publicación en la Revista Española de Investigaciones Sociológicas.

32 Cfr. Richard GUNTHER y Roger BLOUGH, "Conflicto religioso y consenso en España: Historia de dos Constituciones", en Revista de Estudios Políticos, 14, 1980, págs. 12 y ss.

33 Giacomo SANI, Richard GUNTHER y Goldie SHABAD, "Estrategias de los partidos y escisiones de masas en las elecciones parlamentarias españolas de 1979", en Revista de Derecho Político, 11, 1981, págs. 141 y ss.

34 Cfr. Maravall y Santamaria, "Crisis del franquismo", cit., págs. 107 y ss.; también, Manuel RamíRE, "Aproximación al sistema de partidos en España (1931-1981)", en Estudios sobre historia de España, cit., vol. II, págs. 211 y ss.; Jordi CAPO GıL, "Estrategias para un sistema de partidos", en Revista de Estudios Políticos, 23, 1981, págs. 153 y ss.; Josep M. VALLES, «El comportament electoral i la consolidació de la monarquia parlamentaria a Espanya (1977-1981) (amb un post-scriptum obligat per les eleccions de 1982)", en Estudis Electorals/7, cit., págs. 58 y ss. 
los problemas sufridos por sus integrantes resaltaron la fragilidad de la democracia. En palabras de MARAVALL y SANTAMARÍA, dicha fragilidad estaba fundamentalmente ocasionada por «la escasa movilización de apoyo a los partidos, la consolidación aún débil del sistema, la exacerbada competición inter e intrapartidista y la fragmentación del sistema de partidos, que hacía realmente difícil la organización de gobiernos fuertes" 35 . Tras la crisis esporádica del PSOE y la recurrente del PCE, la situación de crisis permanente de UCD profundizó la ineficacia e ineficiencia de sus gobiernos, enfrentados, además, a problemas de envergadura superior. La progresiva descomposición de UCD estuvo, por lo demás, jalonada con procesos electorales en los que sus resultados parecian empeorar irreversiblemente ${ }^{36}$. Los pésimos resultados obtenidos por UCD en las autonómicas de Andalucía, en mayo de 1982, supusieron una anticipación de lo que ocurriría a los pocos meses, cuando se celebraron las elecciones legislativas de octubre de 1982.

\section{EL SEGUNDO CICLO ELECTORAL: ELECCIONES DE «CATACLISMO» (1982) Y ELECCIONES DE «ESPERA» (1986)}

Aunque anunciados en Andalucía y esperados desde hacia tiempo, los resultados de las elecciones de octubre de 1982 no dejaron por ello de ser menos sorprendentes: además de quebrar la, para muchos, firme cristalización del electorado de 1979 , lo hizo en proporciones desconocidas para cualquier país europeo ${ }^{37}$. CACIAGLI ha subrayado que el único

35 Maravall y Santamaria, "Crisis del franquismo", cit., pág. 117.

36 Cfr. Carlos Hunneus, La Unión de Centro Democrático y la transición a la democracia en España. Madrid. Centro de Investigaciones Sociológicas/Sigło XXI, 1985; Richard GUNTHER, "El hundimiento de UCD", en LINZ y MONTERo (eds.), Crisis y cambio, cit., págs. 433 y ss.; Emilio ATtARD Alonso, Vida y muerte de UCD. Barcelona. Planeta, 1983; Jorge DE ESTEBAN y Luis LOPEz GueRRA, Los partidos políticos en la España actual. BarceIona. Planeta/Instituto de Estudios Económicos, 1982, págs. 95 y ss.; CACIAGLI, Elecciones y partidos en la transición española, cit., págs. 258 y SS.; WERT, “El electorado de UCD», cit.; Rafael LOPEZ PINTOR, La opinión pública española del franquismo a la democracia. Madrid. Centro de Investigaciones Sociológicas, 1982, págs. 133 y ss.; y BusE, La nueva democracia española, cit., págs. 72 y ss.

${ }_{37}$ La mayor parte de los capítulos contenidos en LINz y MONTERo (eds.). Crisis y cambio, cit., se dedica al análisis en profundidad de las elecciones de 1982 en sus diversos niveles; cfr. también algunos de los capítulos de PenNiman y MuJaL-León (eds.), Spain at the polls, cit., así como el número monográfico de la Revista Española de Investigaciones Sociologicas, 28, 1984; Alejandro MUÑoz Alonso y otros, Las elecciones del cambio. BarceIona. Argos Vergara, 1984; Rafael LOPEZ PINTOR y otros, Estudio postelectoral sobre la opinión pública española tras las elecciones de octubre de 1982. Madrid. Fundación Juan March, 1983, mimeografiado; Francisco José NaVARRo y otros, Las elecciones generales de 1982. Opiniones y actitudes políticas de los españoles. Madrid. Fundación Friedrich Ebert, 1983; CACIAGLI, Elecciones y partidos en la transición española, cit., págs. 148 y Ss.; BAR, "¿Normalidad o excepcionalidad?", cit., págs. 12 y Ss.; Josep M. VALLÉs, “Las elecciones 
término que por aproximación puede calificarlas es el de "cataclismo", y no le falta razón ${ }^{38}$. Los niveles de participación conocieron una nueva oscilación brusca, aumentando en este caso en cerca de doce puntos porcentuales ${ }^{39}$. La mayor parte de las fuerzas regionales, que constituyeron otro de los rasgos definitorios de las elecciones de 1979, quedó fuera del Congreso. Las agrupaciones de extrema izquierda y extrema derecha obtuvieron apoyos electorales mínimos, hasta el punto de llevarles a su desaparición; la autodisolución de Fuerza Nueva en noviembre de 1982 es paradigmática. La UCD fue, para decirlo gráficamente, triturada por unos electores que reaccionaban asi a las continuas luchas faccionales del partido y a la incapacidad e incoherencia de sus Gobiernos. Sus más de seis millones de votantes en 1979 quedaron reducidos a cerca del millón y medio de 1982; su porcentaje del voto descendió nada menos que del 35 al 7 por 100, y los 168 escaños del hasta entonces partido gobernante se convirtieron en sólo 12. Que se sepa, ningún partido occidental ha sufrido una derrota de esta magnitud; los antecedentes comparados sólo pueden referirse al Partido Republicano Popular de Turquía al final de la década de los cuarenta ${ }^{40}$. Por su parte, el PSOE, con más de diez millones de votos y un 47 por 100 de los mismos, conseguía doblar prácticamente su electorado de 1979 y pasar de 121 a 202 diputados $^{41}$. El triunfo socialista supuso varios hitos de importancia histórica. Por primera vez desde su fundación, el PSOE formaba gobierno en solitario, y por vez primera después de cuarenta años lo hacía un partido de izquierdas. También por vez primera en la historia española un partido obtenía la mayoria absoluta de escaños, un acontecimiento que resulta excepcional, incluso en el contexto de los paises europeos occidentales. La victoria del PSOE significó, por último que, tras sólo cinco años de competencia partidista, España experimentaba la alternancia en el poder, y lo hacia en circunstancias de reforzamiento de la legitimidad que habrian de repercutir muy positivamente en la consolidación de su sistema democrático ${ }^{42}$.

legislativas del 28 de octubre de 1982: Una aproximación de urgencia", en Revista de Estudios Políticos, 33, 1983, págs. 221 y ss.; J. BLONDEL y E. EsEVERRI, «The spanish general election of 1982", en Electoral Studies, 2 (1983), págs. 76 y ss.; Manuel RamlaEz, "El sistema de partidos en España tras las elecciones de 1982", en Revista de Estudios Políticos. 30, 1982, págs. 7 y ss.; R. LOPEZ PINTOR y Manuel JUSTEL, “iniciando el análisis de las elecciones generales de octubre de 1982 (Informe de un sondeo postelectoral)", en Revista Española de Investigaciones Sociológicas, 20, 1982, págs. 155 y ss.; y BuSE, La nueva democracia española, cit., págs. 175 y ss.

38 CACIAGLI, Elecciones y partidos en la transición española, cit., pág. 149.

39 Cfr. José R. MONTERO, "La vuelta a las urnas: Participación, movilización y abs-

tención*, en Linz y MONTERo (eds.), Crisis y cambio, cit., págs. 71 y ss.

$40^{\circ}$ Maravall, La política de la transición, cit., pág. 91.

41 Cfr. Hans-Jürgen PUHLE, "EI PSOE: Un partido dominante y heterogéneo», en LINz y MONTERO (eds.), Crisis y cambio, cit., págs. 289 y ss.

42 Cfr. Maravall y Santamaría, "Crisis del franquismo", cit., págs. 128-129; y SantaMARIA, “Elecciones generales de 1982 y consolidación de la democracia», cit., págs. 9 y ss. 
EI PCE y AP, los dos partidos que flanqueaban a los entonces relevantes, conocieron asimismo suertes electorales contradictorias. La intensificación de la crisis comunista le hizo perder más de un millón de sus antiguos electores y pasar de 23 a 4 escaños. Sus estrategias de crecimiento mediante la atracción de la extrema izquierda y de sectores sustanciales de socialistas se vieron así doblemente truncadas: no sólo ambos objetivos estaban o seguian estando en el PSOE, sino que una parte considerable del propio electorado comunista optó por el PSOE. Reducido a un apoyo electoral y una representación parlamentaria menores que las de los partidos comunistas escandinavos, por ejemplo, el PCE culminó esta etapa fragmentándose en tres partidos comunistas distintos ${ }^{43}$. Por su lado, AP, en coalición con el PDP (una escisión del ala derecha de UCD), encarnaba a la otra cara de la fortuna electoral. Parecian fructificar ahora sus continuos intentos por sustituir la "artificiosidad" de UCD por una "mayoría natural" que compitiese frontalmente con el PSOE en el marco de un bipartidismo no menos "natural»; unos intentos a los que no fueron ajenos, como es sabido, algunos de los denominados poderes fácticos, especialmente los círculos empresariales, y los sectores más conservadores de UCD ${ }^{44}$. AP logró así obtener cinco millones y medio de electores y el 26 por 100 de los votos, quintuplicando con respecto a 1979 el número de sus votantes, cuadruplicando su porcentaje de votos y multiplicando por doce su representación parlamentaria en el Congreso, que pasó de sólo nueve diputados a nada menos que 106 . De forma inversa al fracaso de UCD, el crecimiento de AP es igualmente extraordinario en el escenario europeo desde los años cuarenta: su única equivalencia puede encontrarse en el Rassemblement du Peuple Français, que ascendió de algo menos del 2 al 22 por 100 de los votos a principio de los cincuenta ${ }^{45}$. Aun así, su éxito quedaba relativizado por los más de veinte puntos porcentuales que le separaban del PSOE y por su incapacidad para sustituir plenamente a UCD, sólo una parte de cuyos votantes se dirigieron a la coalición conservadora. Se trataba, pues, de un sub-triunfo, si se admite el neologismo, que demostraba de paso que la verdadera «mayoría natural», contra lo que pensaban los líderes de $A P$, estaba por el momento apoyando al PSOE ${ }^{46}$.

Estos cambio provocaron, de un lado, un descenso apreciable de la fragmentación electoral y parlamentaria. Las dos principales fuerzas políticas, el PSOE y AP-PDP, sumaban cerca del 75 por 100 de los votos y el 88 por 100 de los escaños: por vez primera desde los inicios de la transición, los índices de fragmentación del segundo ciclo conseguían ser

43 Cfr. Richard Gunther, “Los Partidos Comunistas de España", en Linz y MONTERo (eds.), Crisis y cambio, cit., págs. 493 y ss.

44 Cfr. Robert E. MARTINEZ, Business elites in democratic Spain. New Haven. Yale University, mimeografiado, 1984.

45 BLONDEL y ESEVERRI: «The spanish general election of 1982», cit., pág. 72.

46 Cfr. José R. MONTERO, "El sub-triunfo de la derecha: Los apoyos electorales de AP-PDP", en LiNZ y MONTERo (eds.), Crisis y cambio, cit., págs. 345 y ss. 
inferiores a los de las medias europeas ${ }^{47}$. Pero ocasionaron, de otro lado, un espectacular crecimiento de la volatilidad electoral. Siguiendo la pauta observada en 1979, la volatilidad fue relativamente baja entre los bloques de la izquierda y de la derecha, y mucho más intensa en el interior de cada bloque, sobre todo en la derecha.

Según diversos cálculos, la tasa de volatilidad de 1982 sextuplicó la de 1979 y quintuplicó la media europea desde la segunda postguerra $^{48}$. Cerca de siete millones de personas cambiaron su voto de 1979, y otros tres millones pasaron de abstenerse a votar, o votaron tras entrar por primera vez en la edad electoral. En resumen, alrededor del 40 por 100 de los censados modificó su comportamiento electoral inmediatamente anterior ${ }^{49}$. ¿Cómo se distribuyeron estas enormes magnitudes de cambio de voto entre los partidos? Las estimaciones más fiables apuntan a que, en números redondos, los cerca de cinco nuevos millones de votos obtenidos por el PSOE se diversificaban entre un millón procedente del PCE, un millón doscientos mil de UCD y medio millón de otros partidos menores, a los que hay que sumar dos millones de votos de antiguos abstencionistas y de nuevos votantes. Para AP-PDP, el crecimiento electoral fue debido a unos trescientos mil votos de Fuerza Nueva, tres millones de UCD y poco más de un millón de otros partidos regionales y sobre todo de ex abstencionistas ${ }^{50}$. Resultaba así que una tercera parte del electorado del PSOE en 1982 eran votantes a los que cabría denominar «transferidos» por proceder de otros partidos, mientras que la mitad sería “leal» por haber repetido su opción de 1979; la situación es justamente la inversa en el caso de AP-PDP. Esto planteaba a cada uno de ellos problemas específicos en orden a mantener la fidelidad de sus nuevos electores. Las estrategias de conservación de los "votos prestados" en el caso del PSOE, o la de ampliación hacia los votantes de UCD y CDS en el de AP-PDP, se hallan detrás de esas distribuciones.

Aunque no podemos efectuar aquí un análisis detallado de las hipótesis explicativas de los cambios electorales producidos por la consulta de 1982, conviene señalar, al menos, que las motivaciones de los votantes transferidos parecian seguir, en general, una lógica política constituida por una combinación de posiciones ideológicas, respuestas a issues importantes e imágenes positivas o negativas de líderes políticos destacados $^{51}$. Conviene asimismo insistir en que el realineamiento del sistema

47 BAR, “¿Normalidad o excepcionalidad?", cit., págs. 14 y ss.

48 Maravall y Santamaria, "Crisis del franquismo", cit., pág. 226; Bar, "¿Normalidad o excepcionalidad?", cit., págs. 18 y ss.; y VALLÉS, "Las elecciones legislativas de 28 de octubre de $1982 "$, cit., pág. 227.

49 LOPEZ PINTOR y JUSTEL, “Iniciando el análisis de las elecciones generales de octubre de 1982", cit., págs. 156-157; y MARAVALL, La política de la transición, cit., pág. 95.

50 Giacomo SANI, «Los desplazamientos del electorado: Anatomía del cambio», en LINZ y MONTERO (eds.), Crisis y cambio, cit., págs. 1 y ss.

${ }_{51}$ SANI, "Los desplazamientos del electorado", cit., págs. 18 y ss. 
partidista no fue debido a la modificación por los electores de sus posiciones ideológicas moderadas, sino a la crisis del PCE y sobre todo a la desintegración de UCD, que obligaron a sectores numerosos de sus antiguos votantes a reorientar sus preferencias electorales ${ }^{52}$. Dicho de modo distinto, la desaparición del centro y la mayor concentración del voto en el PSOE y (en menor medida) en AP-PDP no eran consecuencias de una radicalización ideológica o actitudinal del electorado, sino de la creencia en la inutilidad del voto a UCD y PCE tras sus largas crisis internas. Manteniéndose constante la moderación ideológica exhibida tiempo atrás por el electorado (y demostrada gráficamente en la continuidad de sus posiciones en la escala izquierda-derecha), el cambio radicaba en la grave disminución de los niveles de credibilidad de las ofertas centristas y comunistas. De ahí que la mayor parte de los antiguos electores de UCD y PCE se refugiara en la lógica de la proximidad espacial para orientar sus nuevas preferencias partidistas: tendieron a votar al partido cuya posición ideológica se percibia más próxima a la ocupada por los propios electores en la escala izquierda-derecha. De ahí también que los antiguos votantes comunistas que transfirieron su voto de 1982 al PSOE sean en líneas generales menos «izquierdistas" que la media de los que escogieron nuevamente al PCE; similarmente, los antiguos votantes centristas que optaron por AP-PDP eran más «derechistas» que los que lo hicieron por UCD o el CDS ${ }^{53}$.

El cambio sufrido por el sistema de partidos impide en rigor, y para empezar, calificarlo de modo que su idoneidad se dé la mano con su durabilidad. No le falta razón a VALLÉs cuando escribe que "nuestros partidos se encuentran, hoy por hoy, a la busca de un sistema", recuerda que un sistema de partidos es "resultado de un proceso histórico de duración relativamente larga» y recomienda, en fin, "una cierta paciencia antes de ceder a la tentación comprensible de caracterizar el sistema español de acuerdo con una de las categorías al uso" ${ }^{54}$. Conviene, sin embargo, salir al paso de dos calificativos muy generalizados. $Y$ es que en numerosos medios políticos o académicos han circulado, como es sabido, conceptuaciones del nuevo sistema de partidos que lo definen como bipartidista y polarizado. Se basaban para ello en la presencia de dos fuerzas mayoritarias, que copaban tres cuartas partes del voto y cerca de nueve de cada diez escaños del Congreso, así como en la considerable distancia ideológica existente entre el PSOE y AP-PDP tras la desaparición de UCD, mayor en cualquier caso de la que había en 1979 entre UCD y el PSOE. A mi juicio, la caracterización del sistema como bipartidista es sencillamente errónea, y la de su polarización exige ciertas matizaciones de importancia. Los más de veinte puntos porcentuales del voto

52 Cfr. GUNTHER, «El realineamiento del sistema de partidos de 1982», cit., págs. 45 y ss.

53 Cfr. SANI, "Anatomía del cambio", cit., págs. 22 y ss.; y MONTERO, "El sub-triunfo de la derecha”, cit., págs. 362 y ss.

54 VALLÉS, "A la busca de un sistema», cit., págs. 152 y 150. 
que separaban al PSOE de AP, al que casi doblaba también en escaños, impide la entrada del sistema español (siquiera sea de forma provisional) en cualquiera de las tipologías sobre el bipartidismo. Esos mismos desequilibrios, a los que puede añadirse la formación de gobiernos homogéneos mayoritarios y la debilidad de los restantes partidos, incluso de los nacionalistas, dificultaba su adecuación a las construcciones del pluralismo moderado. En realidad, el sistema español parecía aproximarse más al de partido predominante democrático, bien que resultara prematuro llegar a esa conclusión después de celebradas unas solas elecciones sobre cuya excepcionalidad existía un acuerdo prácticamente unánime. La provisionalidad atribuida a una parte del voto socialista y la necesaria rearticulación de los espacios del centro y de la derecha habrian de provocar, según se creía, cambios seguros en el formato del sistema de partidos. Sin embargo, la confirmación en 1986 de los resultados de 1982 ha supuesto una nueva sorpresa; y aunque no quepa excluir que la próxima consulta introduzca dichos cambios, una tercera victoria por mayoria absoluta del PSOE podría ratificar el sistema de partidos dominante que aquél protagoniza.

Por lo que hace a la polarización, es cierto que la distancia ideológica entre los partidos relevantes ha aumentado sustancialmente. A esta preocupación se le unían los interrogantes suscitados por la capacidad de AP para intentar, por enésima vez en la historia de la derecha española, la combinación de conservadurismo y democracia, así como para realizar una oposición carente de contenidos ambiguos o semileales sobre el sistema democrático. Esto no obstante, el alcance de la polarización puede relativizarse en atención a varios factores. En primer lugar, y como ya se ha dicho, su crecimiento no fue ocasionado por una brusca radicalización de las opiniones y actitudes del electorado, sino por el realineamiento del sistema de partidos: la desaparición de UCD y su consiguiente reemplazo parcial por AP-PDP no podía, por menos, que aumentar su distancia con respecto al PSOE ${ }^{55}$. Además, la configuración bipolar del nuevo sistema, en el que el vacio de UCD ocasionaba la ausencia de un partido que ocupe las posiciones centrales, incentivaba una competencia interpartidista de naturaleza centrípeta, notablemente reforzada por la distribución unimodal de las preferencias ideológicas de los españoles. Por último, los bajos niveles de apoyo electoral a AP-PDP contribuían, asimismo, a subrayar las tendencias centrípetas del sistema, ya que la única posibilidad de la expansión conservadora, una vez asentada su posición hegemónica en los espacios de la derecha y extrema derecha, radicaba en los espacios centrales del continuo izquierda-derecha ${ }^{56}$.

55 Cfr. Giacomo SANI y Goldie SHABAD, “ ¿Adversarios o competidores?: La polarización del electorado", en LINZ y MONTERo (eds.), Crisis y cambio, cit., págs. 587 y ss.

56 Giacomo SAN y José R. MONTERO, «El espectro político: izquierda, derecha y centro", en Linz y MONTERo (eds.), Crisis y cambio, cit., págs. 155 y ss.; MARAVAll y Santamaria, "Crisis del franquismo", cit., págs. 120-121; y SANTAMARía, “Elecciones generales de 1982 y consolidación de la democracia», págs. 12-13. 
Como se recordará, la principal actividad de los partidos desde 1982 consistió en intentar evitar la repetición de una nueva mayoria socialista. Algunos intentos carecieron de relevancia práctica, otros no llegaron a cristalizar y unos terceros terminaron en un rotundo fracaso. En la izquierda, el PCE quiso finalizar su larga crisis postelectoral mediante un acuerdo con fuerzas políticas de muy distinto signo, que formalizaron la coalición Izquierda Unida (IU) poco antes de la consulta de 1986. Aunque nacida para rentabilizar los cerca de siete millones de noes del referéndum sobre la OTAN, IU obtuvo menos de un millón de votos en junio de 1986: sólo algunos miles más que el PCE en 1982, que la llevaron de cuatro a siete diputados. Mayor interés tuvieron las estrategias adoptadas por los partidos del centro y de la derecha para crear una alternativa a la mayoría socialista. En un primer momento, la obsesión bipartidista de AP le llevó a confiar que podria hacerse con la "mayoría natural» del electorado por sí misma y desde posiciones de derecha: según los cálculos de sus más avisados estrategas, el triunfo electoral conservador se produciría necesariamente tanto por el crecimiento de la abstención como, sobre todo, por la recogida de gran parte de los "votos prestados" del PSOE y de los votos de UCD y CDS. Para ello, AP creó la denominada Coalición Popular con el PDP, con el que había concurrido a las elecciones, y con el Partido Liberal (PL); un grupo virtualmente inexistente. Aunque el PDP y el PL carecían de apoyos electorales propios, su presencia en la CP servia supuestamente para reforzar el encauzamiento de los votos centristas y para amortiguar la imagen conservadora de AP en cuanto principal socio de la Coalición. Sea como fuere, lo cierto es que los objetivos de CP fueron pulverizados en todas las consultas a las que acudió entre 1982 y 1986 . Las desmesuradas -y dificilmente comprensibles- expectativas electorales mantenidas por CP fueron sistemáticamente negadas en las locales y autonómicas de 1983, en las autonómicas vascas y catalanas de 1984 y en la autonómica gallega de 1985. En ninguna de ellas consiguió hacerse, como pretendía, con todo el voto a la derecha del PSOE: las diferencias políticas e ideológicas entre los electorados de centro y de derecha, que los líderes de AP se negaban a reconocer, impidieron, en definitiva, su canalización en un solo partido ${ }^{57}$.

Con posterioridad, las tentativas para la formación de una especie de "gran coalición" antisocialista entre la CP, el PRD, acaso el CDS y con los eventuales apoyos parlamentarios del PNV y de CiU corrieron la misma suerte negativa ${ }^{58}$. Poco antes de las elecciones de junio de 1986 , su principal incógnita residía en los resultados que obtuvieran separadamente la doble alternativa de centro y derecha: "que se mantenga la primacía actual de ésta sobre aquélla, se equilibren ambas o el centro recupere su inicial predominio constituye probablemente el punto de mayor

57 Cfr. MONTERo, «El sub-triunfo de la derecha”, cit., págs. 416 y ss.

58 Cfr. Idem. "Álianza Popular, Coalición Democrática y Coalıción Popular», cit., págs. 53 y ss. 
interés de las elecciones de 1986. Es evidente, a este respecto, que partidos como el CDS o el PRD se sitúan en el plano ideológico mucho más cerca que la Coalición Popular del grueso del electorado que se disputan entre sí. Pero es evidente también que para competir con éxito en ese espacio poseen un grado de credibilidad electoral muy inferior, por el momento, al que suscita AP, como Coalición susceptible de agregar el voto útil» ${ }^{59}$. Esa misma incógnita suponía la mejor prueba del fracaso de los plantamientos estratégicos conservadores, que incluso se reforzaba por sus percepciones deformadas sobre las posiciones ideológicas de los españoles, el atractivo electoral de los socios de la Coalición y la popularidad de sus líderes ${ }^{60}$. Por si faltara algo, la "abstención activa" defendida en el referéndum sobre la OTAN tuvo el raro efecto de compaginar la falta de aciertos con la generación de consecuencias negativas: además de no conseguir ninguno de sus objetivos tácticos en contra del Gobierno socialista, dividió a su propio electorado y suscitó la incomprénsión de los representantes de los sectores sociales a los que la Coalición decía representar ${ }^{61}$.

Si las elecciones de 1982 sorprendieron por la magnitud de sus cambios, las de 1986 lo hicieron por su modestia ${ }^{62}$. Por eso se las ha denominado como una elecciones de "espera" ${ }^{63}$ : los cambios que los analistas preveían para entonces habrán de posponerse hasta 1990, con la decisiva mediación de las elecciones municipales y autonómicas de junio de 1987. En efecto, la consulta arrojó mayores elementos de continuidad que otra cosa. Tras una campaña cuyo principal y casi único motivo pareció residir en la conveniencia de mantener o evitar una nueva mayoría socialista, los resultados electorales agregados (que pueden esconder una gran variación de flujos interpartidistas o una cierta diversidad de los apoyos territoriales) decepcionaron a quienes esperaban grandes varia-

59 SANTAMARIA, "Elecciones generales de 1982 y consolidación de la democracia», cit., pág. 16.

6o Cfr. Richard GunTHER, "The parties in opposition. Prospects for change and for electoral success", en Stanley G. PAYNE (ed.), The politics of democratic Spain. Chicago. Chicago Council on Foreign Relations, 1986), págs. 62 y ss.; y MONTERO, "Los fracasos politicos y electorales de la derecha española", cit., págs. 12 y ss.

61 Cfr. Equipo de Sociología Electoral, «El referéndum de marzo de 1986 sobre la permanencia de España en la OTAN y sus consecuencias sobre el sistema político", en $R e$ vista de Estudios Políticos, 52, 1986, págs. 183 y ss.; y Cesáreo Rodríguez Aguilera, Partidos políticos y referéndum sobre la OTAN", ponencia presentada en la Università de la Pau, San Cugat (Barcelona), julio de 1986.

62 Cfr. Pilar DEL CASTILlo y Giacomo SANI, "Las elecciones de 1986: Continuidad sin consolidación", en Linz y MONTERO (eds.), Crisis y cambio, cit., págs. 625 y ss.; Stanley G. PAYNe, “The elections of june 1986", en PAYNe (ed.), The politics of democratic Spain, cit., págs. 245 y ss.; Joaquín LEgUINA, "La evolución del voto: 1982-1986. España y Madrid», en Sistema, 75, 1986, págs. 113 y ss.; y Juan DIEz NicOLAs, «Análisis y consecuencias de las elecciones generales de 1986", en Cuenta y Razón, 25, 1986, págs. 75 y ss.

63 Tomo esta denominación de las intervenciones de Mario CACIAGLI y Colette YSMAL en la Jornada de análisis que sobre las elecciones de 1986 organizó el Centro de Estudios Constitucionales, Madrid, junio de 1986. 
ciones. Acaso la más notable haya consistido en el descenso de la participación en más de nueve puntos porcentuales, observando así la tónica de las elecciones «normales». Con el 44 por 100 de los votos, el PSOE perdió cuatro puntos porcentuales con respecto a 1982, además de un millón doscientos mil votos y 18 diputados; pero logró mantener la mayoría absoluta de escaños, lo que constituía sin duda un importante éxito. Por el contrario, la CP obtuvo un porcentaje casi idéntico del voto y un número casi igual de escaños (105 en 1986 por 106 en 1982). En comparación con el "sub-triunfo" de la Coalición en 1982, su situación cuatro años después podría, quizás, describirse aplicando el término económico de «estanflación»: la coexistencia de su estancamiento electoral con una creciente inflación de expectativas no auguraba precisamente la estabilidad de la Coalición ni la falta de problemas a AP en cuanto su principal socio. Tampoco IU pareció haber sido capaz de capitalizar demasiados votos del desgaste socialista; como ya se ha visto, las escasas diferencias que le separaban de los resultados del PCE en 1982 patentizaban un evidente fracaso que, por lo demás, no se hubiera posiblemente evitado por la entrada en la Mesa para la Unidad de los Comunistas, de Carrillo, en la IU ${ }^{64}$. En fin, la denominada «batalla por el centro" fue ganada por un CDS que ascendió en más de siete puntos porcentuales, y perdida absolutamente por un PRD que, sobre no conseguir ni un solo escaño, obtuvo menos del 1 por 100 de los votos. El éxito del CDS, que suponia la principal novedad de 1986, fue, sin embargo, menor de lo que parecía a primera vista: el 9 por 100 de votos conseguido le permitía contar con grupo parlamentario propio y crecer como partido, pero no significaba una modificación radical del espacio del centro, habida cuenta de que la suma del voto CDS y UCD de 1982 coincidía con la de CDS cuatro años después.

En cuanto segundas elecciones del ciclo, las de 1986 han permitido la permanencia de muchas de las características presentes en las de 1982. Se ha seguido manteniendo la rigidez de transferencia de votos entre los bloques de izquierda y derecha y la relativa fluidez de los alineamientos políticos, especialmente, como en 1979, en el de la derecha. Aunque la presencia del CDS ha debido aumentar la fragmentación parlamentaria, la distribución de votos y escaños entre las dos principales fuerzas no ha sufrido (hasta la ruptura de Coalición Popular, que ha reducido a AP a sólo 68 diputados) grandes variaciones. El escaso cambio en términos de votos ha ocasionado una tasa de volatilidad baja, y permitido la continuidad del desequilibrio entre los partidos de izquierda y de derecha. En fin, el sistema de partidos tampoco ha conocido una modificación sustancial en términos de su polarización, de la necesaria competencia centripeta y de sus integrantes: el PSOE sigue apareciendo como partido dominante y contando con mayoría absoluta para apoyar gobiernos ho-

64 Del Castillo y Sani, “Las elecciones de 1986", cit., págs. 637 y ss. 
mogéneos, sin que pueda inquietarle una oposición de la que le alejan 18 puntos porcentuales, la pequeña representación comunista a su izquierda o el 10 por 100 aproximadamente del CDS.

\section{PERSPECTIVAS FUTURAS: DE NUEVO, LA INCERTIDUMBRE (RELATIVA)}

¿Qué posibilidades hay de que esta continuidad del voto pueda mantenerse en un futuro inmediato? En mi opinión, no demasiadas. ¿En qué sentido cabe prever su evolución? La pregunta resulta, por el momento, de imposible contestación. Apurando la prudencia, la única respuesta que cabe dar es la de que nos encontramos a las puertas de un nuevo ciclo en la trayectoria electoral de los partidos, pero con tantas incógnitas como para no aventurar más previsiones. La lectura que un sector de la élite política hizo de las elecciones de 1986 ha alterado de forma importante el panorama de los partidos, originando nuevas dosis de incertidumbre sobre el formato futuro del sistema. $Y$ los resultados de la triple convocatoria de junio de 1987, pese a su naturaleza de elecciones de "segundo orden" ${ }^{65}$, o acaso por eso mismo, no hacen sino avivarlas debido a la transferencia de un sector de antiguos votantes socialistas hacia partidos que han logrado hacerse presentes en algunos Parlamentos regionales y municipios de indudable significación. La ruptura de $\mathrm{CP}$, la grave crisis de AP ocasionada por la dimisión de su, hasta entonces, máximo líder y su competencia futura con el CDS introducen dudas sobre la capacidad conservadora para llegar incluso a sus "techos" electorales de 1982 y 1986. La posición estratégica ocupada por el CDS se ha revalidado en las elecciones de 1987, pero su pretensión de mejorarla en 1990 no está por completo garantizada en una consulta de naturaleza distinta y con diferentes mecanismos de motivación del voto. Es ciertamente seguro que se reanuden los intentos para recrear una mayoría alternativa al PSOE, pero sus componentes siguen estando sujetos a demasiados interrogantes. Es posible, tras el fracaso obtenido en dos elecciones legislativas sucesivas, que AP haya perdido la ocasión para protagonizar esa alternativa. Sus evidentes dificultades para expandirse hacia el centro y los amplios rechazos que genera, por no hablar de unos supuestos estratégicos tan desmesurados como irreales ${ }^{66}$, hacen aparentemente inviable que AP obtuviera, incluso, una tercera parte de los votos y se convierta en eje de una coalición alternativa a un PSOE mino-

65 Karl REIF, "Ten second order national elections", en K. REIF (ed.), Ten european elections. Aldershot. Gower, 1985, págs. 10 y ss. págs. 20 y ss. 
ritario ${ }^{67}$. Es más que dudoso que de esa coalición forme parte principal un CDS reforzado, mientras que resulta más fácil imaginar al CDS coaligado con un PSOE minoritario. Parece igualmente descartada la reproducción de la coalición de AP con el PDP tras las heridas abiertas por la ruptura unilateral de los democristianos y sobre todo por la reciente constatación de sus débiles apoyos electorales, y tampoco parece factible que a ella se sume el CDS. Y suena sobre todo a ciencia-ficción, en fin, una coalición entre el CDS, el PDP y los partidos nacionalistas, siempre en el más que improbable supuesto de que el PDP logre hacerse un sitio en el congestionado espacio existente entre el PSOE, el CDS y AP 68 .

Como en los años iniciales de la transición, la articulación política de la derecha sigue siendo así, diez años después, un problema a resolver. Pero su solución no depende tanto de unos electores cuya moderación ideológica no ha sufridos cambios apreciables, cuanto de la élite política conservadora, cuya notable capacidad de autodestrucción e inadecuación a la política competitiva de partidos no parece haber finalizado. A las puertas de un tercer ciclo en la evolución electoral de los partidos; su principal incógnita sigue residiendo, como en los dos anteriores, en la forma de articulación del centro-derecha y derecha durante los próximos años. Una incógnita cuya solución dependerá, en definitiva, del aprovechamiento de las posiciones regionales y locales ocupadas por el CDS tras las últimas elecciones, de la capacidad de AP para evitar errores pasados sobre su espacio óptimo de competición, a la búsqueda de esa inexistente «mayoría natural» conservadora, y, naturalmente, de la habilidad del PSOE para seguir manteniendo su excepcional situación en el sistema de partidos. 